\title{
Admission and Survival Trends in Hedgehogs Admitted to RSPCA Wildlife Rehabilitation Centres
}

\author{
Nicolette Dowler Burroughes ${ }^{1}$ (D) Jonathan Dowler ${ }^{2} \cdot$ Guy Burroughes $^{3}$
}

Received: 3 February 2020/Revised: 8 February 2021 / Accepted: 6 March 2021 / Published online: 1 April 2021

(C) The Author(s) 2021

\begin{abstract}
The hedgehog (Erinaceus europaeus) population is in decline in the UK and they are the most frequently admitted mammal to British Wildlife Rehabilitation Centres (WRCs). Whilst successful, UK rehabilitation is timeconsuming and expensive and few large-scale studies into UK WRC admission and survival rates have been published in the last decade. This paper examines admission and survival trends in 19,577 hedgehogs admitted to Royal Society for the Prevention of Cruelty to Animals centres over a 13 year period (2005-2017) to gauge the state of Britain's hedgehogs in WRCs and to gain indirect insight into the wild population. During the studied period, admissions more than doubled. Admission weights were greater in later than early litter juveniles. The survival improved $26 \%$ overall, and $33 \%$ in juveniles. Twenty two percent of animals died or were euthanased within $48 \mathrm{~h}$ of admission. Kaplan-Meier analysis gave survivor functions of 0.78 at 2 days, 0.66 at 10 days, 0.62 at 20 days, and 0.53 at 80 days. Survival was independent of admission weight in each age category. In particular, survival was greater in early litter juveniles than in adults or late litter juveniles; and across the breeding season diminished in juveniles and increased in adults. These data suggest factors impacting hedgehog survival have remained stable despite population decrease; that care methods have improved; and that late litters are more vulnerable than early. For WRCs this
\end{abstract}

Nicolette Dowler Burroughes

nicolette.dowler@1mh.ox.ac.uk

1 University of Oxford, Oxford OX1 3SZ, UK

2 Knyftons Farm, Westbury-sub-Mendip, Somerset BA51HN, UK

3 Blakes Barn, Kingston Road, Abingdon OX13 5NX, UK reaffirms that current methods are successful, but that further resources could be directed towards late litters.

Keywords Hedgehog - Wildlife rehabilitation - KaplanMeier · Hedgehog survival · Erinaceus europaeus

\section{Introduction}

The number of hedgehogs in Great Britain has been steadily declining over the last few decades (Hof 2009; Roos 2012; Wilson 2018). Additionally, hedgehogs are the most frequently admitted mammal to British Wildlife Rehabilitation Centres WRCs (Kirkwood 2003; Bullen 2002; Molony 2006), where they require care for a number of issues such as malnutrition, traumatic injury, disease, parasite burden and orphaning (Robinson and Routh 1999; Bunnell 2001; Stocker 2013); many of which are the result of human influence (Reeve and Huijser 1999). The care for hedgehogs in WRCs is time-consuming and expensive but has the end goal of rehabilitating them and releasing them healthy back into the wild (Stocker 2013; Mullineaux 2014, RSPCA). There has been some research into the post release success of hedgehogs suggesting that it is largely successful (Morris et al. 1994; Sainsbury et al 1996; Reeve 1998; Molony 2006; Warwick et al 2006; Yarnell et al 2019). Although there have been studies into rescue centres' admission data and mortality rates in previous decades (Molony 2007; Grogan 2013) and other countries (Martinez 2014), there has only been limited research on British centres for the past decade, and what there is limited geographically and in its numbers.

West European Hedgehogs (Erinaceus europaeus) are small insectivorous mammals common to the British countryside. They live on a diet of macro-invertebrates, 
and are nocturnal, foraging for food at night and sleeping during the day in nests (Reeve 1994). From late spring to autumn they breed and have up to 21 , looked after solely by the female (Deanesley 1934; Reeve 1981; Jackson 2006). The litters have between 2 and 6 young, with an average of 4.4 (Morris 1977), and they are milk fed for the first 6 weeks of their lives until they are sufficiently independent to forage outside the nest, first with their mothers, and then on their own (Morris 1977). Hedgehogs hibernate in nests over the winter months, using fat reserves built up over the summer to sustain them (Reeve 1994). The natural mortality of wild hedgehogs is high (Reeve 1994); and believed to reach $20 \%$ before weaning (Morris 1977), with approximately only $2 / 3$ rds surviving their first year (Kristiansson 1990; Yarnell et al 2019).

In previous studies, success has been defined by release rates. However, this has disadvantages; no information can be provided regarding animals which die in the wild, some animals admitted to WRCs would have survived in the wild, and some animals survive through the intervention of the WRC which would otherwise have died. Kaplan-Meier survival analysis is the statistical technique used to address incomplete data of this type, but has not hitherto been used in examining data from WRCs. WRCs have the potential to provide large sample sizes, and detailed information on causation and mortality. Despite this only 2 large-scale UK studies of this type have been published, one using 2000-2004 data from Royal Society for the Prevention of Cruelty to Animals centres (RSPCA) (Molony 2007), and the other using 1992-1998 British Wildlife Rehabilitation Centres (BWRCs) data from 2 UK centres and one from the Netherlands (Reeve and Huisjer 1999).

In this paper we examine admission and survival trends in hedgehogs admitted to RSPCA WRCs over a 13 year period, substantially the largest published sample. We use Kaplan-Meier survival analysis to provide robust estimates of mortality. It is hoped that that these data will aid the optimum allocation of resources within WRCs, and to give indirect insight into events in the wild.

\section{Methods}

The following outlines the protocol used to analyse data collected from RSPCA centres between 2005 to 2017, in sufficient detail to allow Kaplan-Meier survival analysis, Comparisons between adults, early litter juveniles and late litter juveniles, and limited exploration of relationships between causation and mortality.

Admission procedure at the RSPCA rescue centres involves collecting key data upon the animal's arrival (unless urgent treatment is required). These include the extent of injury or illness, where the animal was found, weight, sex, whether the animal is juvenile or adult, and any record of previous treatment. Animals are then assessed by trained staff, who decide, based on guidance by the RSPCA's protocol, whether to euthanase, treat, or return immediately to the wild. Animals with severe injury, illness, parasite infestation, and the very premature young may all be considered for euthanasia. Treatment algorithms are standardized across centres and administered by trained wildlife staff as well as veterinary staff who undertake any necessary procedures (RSPCA 2013).

Data obtained from the RSPCA were:

- Date of Admission

- Weight on admission

- Date of release or death

- Whether released or died

- Admission reason

If a specific reason for admission was given, it was categorised as trauma, disease, malnutrition, or orphan, grouped as follows:

- Trauma—including injury, caught/entangled, fishing litter, garden injury, collision

- Malnutrition-including starvation and weakness

- Orphan—orphan, inexperienced juvenile.

Admission groups were defined as follows; adult if admission weight was $>250 \mathrm{~g}$, and juvenile if $<250 \mathrm{~g}$, $250 \mathrm{~g}$ being the weight at which most juveniles are considered weaned, and will no longer be milk fed (RSPCA 2013; Bunnell 2009; Bexton 2016). Adults and sub-adults could not be distinguished on the basis of the available data. Weekly admission figures for juveniles were obtained, and 2 admission peaks identified (Fig. 1), on the basis of which animals admitted during the months of June,

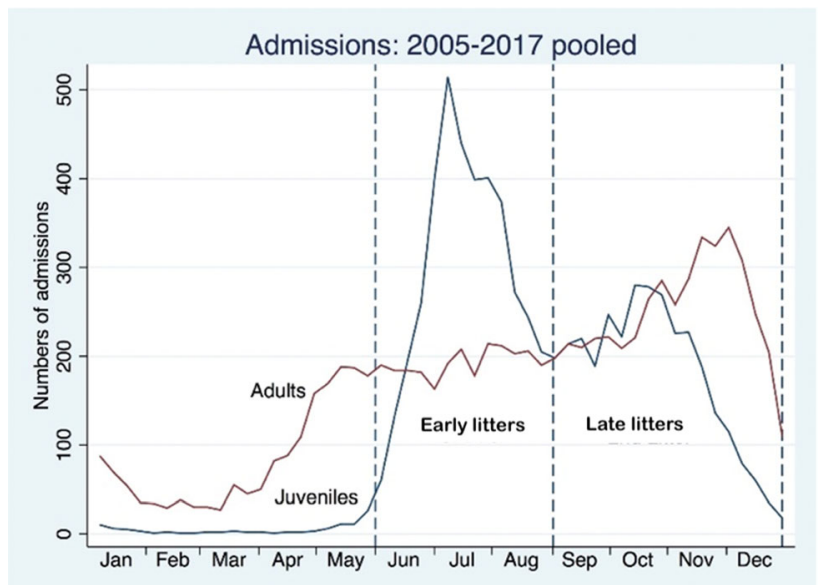

Fig. 1 Pooled admission distribution across the year of adults and juveniles, showing a bimodal distribution in juveniles across the study period, the first being designated 'early litters' and the second as 'late litters' 
July and August were designated early litter, and those admitted in September, October, November, and December as late litter. The timings of admission peaks were recorded for each year.

The study examines only animals admitted during the breeding season, since these make up the majority of admissions, and factors leading to admission outside the breeding season are likely to be different to those within it. Animals were excluded where the record was inaccurate or incomplete, and where the animals died or were euthanased before admission was complete. The resulting sample consisted of 13,907 animals, made up of 6848 adults, 4001 early litter juveniles, and 3058 late litter juveniles. The admissions per year in each category are depicted in Fig. 1.

The outcome variable was survival to release, which was examined in relation to adults versus juveniles, early litter versus late litter, and admission weight.

\section{Statistical Methods}

Means of 2 groups were compared using Students t-test. Pearson's chi-squared test was used to compare proportions. Least-squares linear regression was used to examine the effect of independent variables on continuous dependent variables.

Because diagnostic groups were derived from a limited, nonrandom subset of the data, only limited quantitative statements were made about them, and they were not included in multivariate analysis. Separate Mantel-Haenszel tests were used to compare survival in animals within each diagnostic group with those not allocated to a diagnostic group.

The Kaplan-Meier estimator allows the estimation survival function from lifetime data. An important advantage of this curve is that it can take into account some types of censored data, particularly right-censoring, which occurs if an animal withdrawn from the study or the data is corrupted or lost. If the survival functions between two or more groups are to be compared, the third piece of data supplied was the group assignment of each animal. In this case, the data used is date in the year of initial entry until withdrawal or death, against the proposed populations.

Cox's proportional hazards regression, weighted where appropriate for size-related sampling differences, was used to examine survival by month and year of admission, and admission group. Since admission weight interacts with admission group, the effect of admission weight on survival in each group was examined using a separate regression. Admission weights in animals $<80 \mathrm{~g}$ were stratified for survival analysis to determine a minimum viable survival weight.

A $p$-value of $<0.05$ was adopted for statistical significance. Values are given with $95 \%$ confidence intervals in brackets after them. All statistical analysis was carried out using Stata 15.1, Statacorp Texas.

\section{Results}

\section{Admissions}

The aggregate weekly admissions of the juveniles showed a bimodal distribution with a high narrow peak from June to August, and a second broader peak from September to December (Fig. 1). Adults showed a gradual increase in numbers over the breeding season. Yearly admissions more than doubled (60 animals, $5.78 \%$ per year) over the period studied (Fig. 2), rising from 666 in 2005 to 1481 in 2017. Admissions increased proportionately in adults, early and late litter animals. There was no change in admission weights over the period studied. Mean admission weights were $452 \mathrm{~g}$ for adult animals, $126 \mathrm{~g}$ for early litters, and $181 \mathrm{~g}$ for late litters, thus $44 \%$ greater in late than in early litters.

Admission reasons sufficiently specific were found in $57 \%$ of animals, and were grouped into four broad categories: trauma, orphan, malnutrition, and disease, distributed as follows (Table 1):

Over the breeding season, trauma admissions declined, particularly in adults, and malnutrition admissions increased in both juveniles and adults.

\section{Survival}

Kaplan-Meier survivor functions (the probability of surviving at a given time point) were (Table 2):

The survival curves generated by these functions in early litters, late litters and adults are represented in Fig. 3.

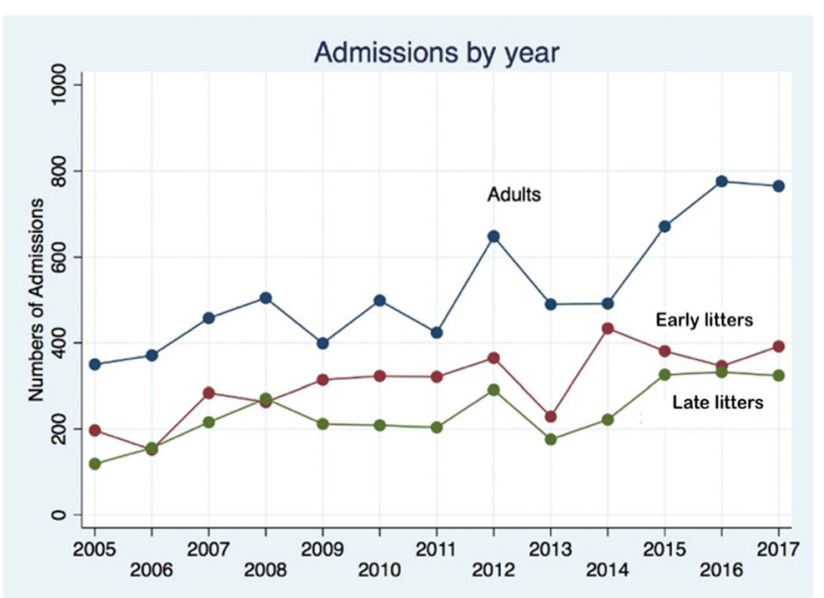

Fig. 2 Relative admissions of adults, early litters, and late litters over the study period 
Table 1 Cause of admission across different age groups

\begin{tabular}{lcccc}
\hline & Trauma $(\%)$ & Disease $(\%)$ & Malnutrition $(\%)$ & Orphan (\%) \\
\hline Adult & 46 & 10 & 44 & - \\
Early litter & 10 & 3 & 3 & 84 \\
Late litter & 9 & 3 & 50 & 38 \\
\hline
\end{tabular}

Table 2 Kaplan-Meier survivor functions at given time points for different age groups

\begin{tabular}{lllll}
\hline & 2 days & 10 days & 20 days & 80 days \\
\hline Adult & 0.75 & 0.63 & 0.60 & 0.51 \\
Juveniles & 0.81 & 0.69 & 0.64 & 0.56 \\
Early litter & 0.84 & 0.72 & 0.67 & 0.58 \\
Late litter & 0.77 & 0.64 & 0.60 & 0.52 \\
Overall & 0.78 & 0.66 & 0.62 & 0.53 \\
\hline
\end{tabular}

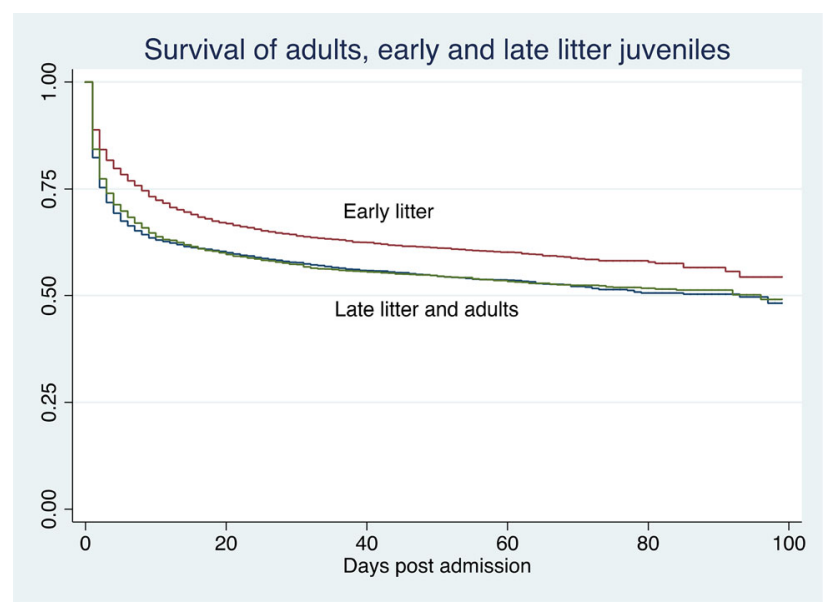

Fig. 3 Kaplan-Meier survival curves of early litter versus late litters and adults

There was $2.2 \%$ improvement in all-group survival in year on year basis, and $26 \%$ overall improvement during the study period (Hazard ratio 0.978: 0.971, 0.984)). Survival was greater in early litters, and less in adult and late litter animals (HR 1.27:1.20,1.35, and HR 1.25: 1.16,1.34) Survival improved $1.8 \%$ per year $(21 \%$ over the study period) in adults, and $3.1 \%$ per year (33\% over the study period) in juveniles. In adults, survival improved $12 \%$ per month over the breeding season, but in juveniles it deteriorated by $10 \%$ over the breeding season. Compared to animals with no diagnostic group allocated, survival was the same in trauma admissions, and better in orphans and malnourished.

Mean admission weight did not influence survival in any admission category. In animals weighing under $80 \mathrm{~g}$ (strata 15-40 g, 41-60 g, 61-80 g), survival declined with diminishing weight, but even 15-40 g animals had a $41 \%$ 80-day survival, and no minimum viable weight was identified.

\section{Discussion}

\section{Admissions}

Hedgehog admissions increased over the period studied in all admission groups. The overall increase was $2.4 \%$ per year, similar to a $2.86 \%$ annual increase between 1996 and 2017 observed in data from the Jersey Hedgehog Preservation Group (N Reeve-personal communication, Molony 2007). These figures are comparable to the simultaneous rate of population decline (Roos et al 2012), and probably represent different expressions of the same threats to hedgehog survival. The increase in admissions might be due to improved public awareness of the plight of the hedgehog, but if this were so, one might expect that over time, animals would be admitted at an earlier stage, with fewer dead or moribund on arrival, higher admission weights and reduced 48-h mortality, none of which proved to be the case.

Because admission diagnoses in this study represented a limited, non-random subset of animals, they must be interpreted with caution. With due allowance for this, proportions of animals diagnosed with trauma were unchanged over the study period in adults, early and late litter juveniles. These proportions, however, only relate to sub-lethal injury, and thus may not reflect population loss through lethal trauma such as roadkill (Wembridge et al. 2016). Trauma admissions diminished across the breeding season, almost exclusively in adults, perhaps because the ranging behaviour of male hedgehogs lessens (Reeve 1982). Admissions for malnutrition increased during the study period, perhaps reflecting progressive habitat changes and reduced food availability (Hof and Bright 2009). Malnutrition appeared mainly to affect adults, including postpartum females which may be emaciated (Jackson 2006), and late litter juveniles, both having to gain weight rapidly to survive hibernation (Morris 1984; Bunnell 2009), which is consistent with the increase in malnutrition admissions across the breeding season.

Admissions of juveniles showed a bimodal distribution during the breeding season, with a higher narrower peak (weeks 26-31) in June July and August, followed by a 
lower, wider peak (weeks 39-46) in September, October, November and December. There was no change in the timing of admission peaks for early and late litters over the 13 year study period. This is perhaps too short a period to detect the possible phenological effects of climate change (Jackson 2006).

Median peak admission of early litters in early July (week 27-28) fits with previous research (Jackson 2006; Bunnell 2009). In the later litters, median peak admission of early October (Week 40) correlates with Bunnell's finding of mid-October, but differs from findings in the Hebrides (Jackson 2006), where the latest births recorded were in mid-September, resulting in the latest estimated achievement of this weight to be mid-October, in contrast to the latest found here at the end of November. This may be due to the different summer length between England (the location of RSPCA WRCs) and the Hebrides and would support research that claims breeding is triggered by sustained periods of warmth (Fowler 1988; Reeve 1994). Summer is considerably shorter in the Hebrides, and to have any hope of having successful late litters whether as 1 st litters, or 2 nd, they must breed much earlier on. This is supported by research suggesting that 2 nd litters are not attempted in Sweden, which has even shorter summers (Kristiansson 1981).

Admission weights were higher in late than early litters, consistent with the observations of Bunnell (2009). Orphans were commoner in early than late litters $(77 \%$ versus $23 \%$ ), but malnourished were commoner in late litters (42\% vs $4 \%$ ). This may be as orphans admitted following nest disruption (and so unweaned) are likely to be younger and lighter, whereas if the orphans are old enough to be part-weaned and have begun to forage on their own (Reeve 1994; Bunnell 2009), failure to successfully feed themselves might cause the malnutrition which ultimately brought them into the WRCs. The mean admission weight for late litters was $181 \mathrm{~g}$, which although generally lower than one would hope to see in a weaned hedgehog, is consistent with an underweight juvenile.

In both litters, 95\% confidence intervals for admission weights span only $4 \mathrm{~g}$, and the difference between means is substantial (65 g), supporting representation of the 2 litters as discrete entities. 95\% confidence intervals in adult admission weights spans only $9 \mathrm{~g}$. The weight difference between the litters is statistically significant (44\%, $p<0.00005$ ) and along with the poorer survival of the later litters suggests possible specific developmental stages with increased vulnerability in juvenile hedgehogs, where the timing of these stages during the year may be critical.

There are several possible explanations for the admission weight and survival differences between early and later litters. It may be that a proportion of late litters may be a second attempt to breed after a failed early litter, or a 1st litter for some young females maturing later in the Summer (Reeve 1994). Jackson (2006) also noted that the reasons for failed breeding attempts were mostly unknown but observed two attempts (13\% of known failures), both in the early season, which failed prior to birth. On two other occasions the mother died during the lactation period, apparently from exhaustion. Additionally, it might be argued that if the 2nd litter follows a failed 1st, the female may have an underlying health issue or weakness causing her 1st litter to fail but in the 2nd litter does not appear or develop until later, thus causing the eventual mortality of orphans. Likewise, if a 2nd litter follows a successful 1st, the female's resources may be depleted, and she is unable to give the 2nd litter the same support. Hedgehog milk is full of crucial antibodies and passes on passive immunity (Landes et al 1998; Morris 1961) so if not fully supplied the 2nd litter would be weaker and more vulnerable, and so less likely to survive despite intervention from Rescue Centres.

\section{Survival}

In Reeve and Husijer's study (1999), the percentage of animals released ranged from 40 to $75 \%$, in the Jersey study 67\% (N Reeve-personal communication, Molony 2007; Reeve and Huijser 1999), and in our study, 58\%. If animals surviving less than $48 \mathrm{~h}$ are excluded, Molony reports a $53 \%$ release rate, our figure being $78 \%$. These figures are broadly comparable, and compare favourably with release rates, across all species, of $42 \%$ in RSPCA centres, and 40\% in BWRC centres (Grogan and Kelly 2013). Given recent research into the survival overwinter of WRC releases compared to wild animals found it to be roughly equal (Yarnell et al. 2019), this is very encouraging, and suggests that the guidelines on release set by the RSPCA are successful.

Survival increased by $2.2 \%$ per year, $(26 \%$ over the study period), particularly in juveniles, in which survival increased by $36 \%$. This did not appear to be due to improvement in the condition of animals on admission; there was no change in proportions of animals dead or moribund on arrival, in admission weight, or in 48-h mortality over the study period. Nor did it appear to be due to progressive change in the proportions of adults, early litters, and late litters, since this was unaltered during the course of the study. Increased malnutrition admissions, associated with better survival, may have made a contribution. Without any other obvious factors, it appears that care at the centres is more successful. The RSPCA protocol has not significantly changed during the study period, however the individual experience and expertise of the staff there can only have improved, especially as wildlife care is a relatively recent field. 
Reeve and Huijser report causation and seasonal variation similar those in our sample with deaths due to "unnatural causes" such as trauma predominating in the early part of the breeding season, and due to "natural causes" including malnutrition in the later part, and similar patterns were seen in the Jersey study (Reeve-personal communication, Molony 2007; Reeve and Huijser 1999). In our study, survival was greater in early litters than in late litters and adults. Orphan admissions were commoner in early than late litters (77\% versus $23 \%$ ), malnutrition admissions commoner in late than early litters $(42 \%$ versus $4 \%)$ and commoner still in adults 53\%. Late litter animals have to sustain a higher growth rate than early litters (Bunnell 2009) with a diminishing food supply, and thus being perhaps in a less healthy condition than orphans, be less likely to survive. The view that there is a high mortality in late litters related to an inability to reach adequate hibernation weight has been challenged by Bunnell (Bunnell 2009), but the higher mortality of late litters in our sample, and their tendency to malnutrition tend to support it. In adults, postpartum females are vulnerable to malnutrition (Jackson 2006) which would also explain the increased malnutrition later in the year. Likewise, trauma admissions, with relatively poorer survival, were commonest in adults (73\% in adults, $27 \%$ in juveniles), which is consistent with their expected greater motility, thus greater exposure to unnatural threats.

Within admission groups, survival was independent of admission weight, endorsing Molony's findings (Molony 2007). The tight clustering of admission weights around the mean, however, makes it less likely that such an association would be found. It is encouraging to those inclined to resuscitate animals of very low weight, that even those below $40 \mathrm{~g}$ had reasonable survival rates, but it is likely that some selection bias is present, especially as those arriving at very low weights and in poor condition are more likely to be euthanised within $48 \mathrm{~h}$ in accordance with RSPCA protocol.

Mortality of adult animals diminished across the breeding season. This may reflect the diminishing effect of trauma, which declines as the year goes on, as male adults cease to roam in search of mates (Reeve 1982). Mortality of juveniles increased throughout the breeding season. This may reflect a transition from early litter orphan admissions with better survival to late litter malnutrition admissions associated with higher mortality.

\section{Conclusion}

This study shows a substantial improvement in survival of admitted animals over time. These results demonstrate the success of current methods of care and treatment at the
RSPCA, particularly in early litters and adults. The relatively poorer survival of later litters would be a useful area for further study.

Robust evidence is presented of a bimodal population of juveniles over the breeding season. This appears to support arguments that a proportion of hedgehogs in Britain have 2 litters, though the evidence is indirect.

The most intriguing finding is that admissions were found to have increased substantially over time despite simultaneous decreases in overall population. Greater public awareness of WRCs, and increased proximity of hedgehogs to populated areas due to habitat loss are possible explanations, but further study is required.

Acknowledgement We would like to acknowledge and thank the RSPCA for supplying us with this data.

Open Access This article is licensed under a Creative Commons Attribution 4.0 International License, which permits use, sharing, adaptation, distribution and reproduction in any medium or format, as long as you give appropriate credit to the original author(s) and the source, provide a link to the Creative Commons licence, and indicate if changes were made. The images or other third party material in this article are included in the article's Creative Commons licence, unless indicated otherwise in a credit line to the material. If material is not included in the article's Creative Commons licence and your intended use is not permitted by statutory regulation or exceeds the permitted use, you will need to obtain permission directly from the copyright holder. To view a copy of this licence, visit http://creativecommons.org/licenses/by/4.0/.

\section{References}

Bexton, S. 2016. Hedgehogs. In BSAVA manual of wildlife casualties, Chapter 12, 117-136. British Small Animal Association (BSAVA) Library.

Bullen, K. 2002. Hedgehog rehabilitation: A guide to the rescue, rearing, nursing, care, diagnosis, treatment, welfare and release of the Hedgehog Erinaceus europaeus. British Hedgehog Preservation Society.

Bunnell, T. 2001. The importance of faecal indices in assessing gastrointestinal parasite infestation and bacterial infection in the hedgehog (Erinaceus europaeus). Journal of Wildife Rehabilitation 24: 13-17.

Bunnell, T. 2009. Growth rate in early and late litters of the European hedgehog (Erinaceus europaeus). Lutra 52: 15-22.

Deanesley, R. 1934. VI. The Reproductive processes of certain mammals. Part VI.-The reproductive cycle of the female hedgehog. Philosophical transactions of the royal Society of London. Series B, containing papers of a biological character, 223, 494-508, 239-276.

Doncaster, C.P. 1994. Factors regulating local variations in abundance: field tests on hedgehogs, Erinaceus europaeus. Oikos 69: 182-192.

Fowler, P.A. 1988. Seasonal endocrine cycles in the European hedgehog, Erinaceus europaeus. Reproduction 84: 259-272.

Grogan, A., and A. Kelly. 2013. A review of RSPCA research into wildlife rehabilitation. Veterinary Record 172: 211-215. 
Guy, A.J., D. Curnoe, and P.B. Banks. 2013. A survey of current mammal rehabilitation and release practices. Biodiversity Conservation 22: $825-837$.

Hof, A.R. 2009. A study of the current status of the hedgehog (Erinaceus europaeus), and its decline in Great Britain since 1960. Doctoral dissertation, University of London.

Hof, A.R., and P.W. Bright. 2009. The value of green-spaces in builtup areas for western hedgehogs. Lutra 52: 69-82.

Hof, A.R., and P.W. Bright. 2010. The value of agri-environment schemes for macro-invertebrate feeders: hedgehogs on arable farms in Britain. Animal Conservation 13: 467-473.

Jackson, D.B. 2006. Factors affecting the abundance of introduced hedgehogs (Erinaceus europaeus) to the Hebridean island of South Uist in the absence of natural predators and implications for nesting birds. Journal of Zoology 271: 210-217.

Johnson, H., and E. Thomas. 2015. Guidance for detecting hedgehogs using footprint tracking tunnels. People's Trust for Endangered Species (PTES) and the Hedgehogs Preservation Society, Version May 12015.

Kirkwood, J.K. 2003. Introduction: Wildlife casualties and the veterinary surgeon. In BSAVA manual of wildlife casualties, ed. E. Mullineaux, D. Best, and J.E. Cooper, 1-5. Gloucester, MA: BSAVA Publications.

Kristiansson, H. 1981. Distribution of the European hedgehog (Erinaceus europaeus L.) in Sweden and Finland. In Annales Zoologici Fennici, 115-119. Finnish Academy of Sciences, Societas Scientiarum Fennica, Societas pro Fauna et Flora Fenn.

Kristiansson, H. 1990. Population variables and causes of mortality in a hedgehog (Erinaceous europaeus) population in southern Sweden. Journal of Zoology 220: 391-404.

Landes, E., J. Zentek, P. Wole, and J. Kamphues. 1998. Investigation of milk composition in hedgehogs. Journal of Animal Physiology and Animal Nutrition 80: 179-184.

Martínez, J.C., A.I. Rosique, and M.S. Royo. 2014. Causes of admission and final dispositions of hedgehogs admitted to three Wildlife Rehabilitation Centers in eastern Spain. Hystrix, the Italian Journal of Mammalogy 25: 107-110.

Molony, S.E., P.J. Baker, L. Garland, I.C. Cuthill, and S. Harris. 2007. Factors that can be used to predict release rates for wildlife casualties. Animal Welfare 16: 361-367.

Molony, S.E., C.V. Dowding, P.J. Baker, I.C. Cuthill, and S. Harris. 2006. The effect of translocation and temporary captivity on wildlife rehabilitation success: An experimental study using European hedgehogs (Erinaceus europaeus). Biological Conservation 130: $530-537$.

Morris, B. 1961. The transmission of anti-Salmonella agglutinins from the mother to the young in Erinaceus europaea, with some observations on the active immunization of suckling hedgehogs. Proceedings of the Royal Society of London. Series B Biological Sciences 154: 369-376.

Morris, P. 1977. Pre-weaning mortality in the hedgehog (Erinaceus europaecus). Journal of Zoology 181: 162-164.

Morris, P. 1984. An estimate of the minimum body weight necessary for hedgehogs (Erinaceus europaeus) to survive hibernation. Journal of Zoology, London 203: 291-294.
Morris, P.A., and H. Warwick. 1994. A study of rehabilitated juvenile hedgehogs after release into the wild. Animal Welfare 3: $163-177$.

Mullineaux, E. 2014. Veterinary treatment and rehabilitation of indigenous wildlife. Journal of Small Animal Practice 55: 293-300.

Reeve, N. 1994. Hedgehogs. London: T \& AD Poyser. Ltd.

Reeve, N.J. 1981. A field study of the hedgehog (Erinaceus europaeus) with particular referance to movements and behavior. Doctoral dissertation, University of London.

Reeve, N.J. 1982. The home range of the hedgehog as revealed by a radio tracking study. Symposia of the Zoological Society of London 49: 207-230.

Reeve, N.J. 1998. The survival and welfare of hedgehogs (Erinaceus europaeus) after release back into the wild. Animal Welfare 7: 189-202.

Reeve, N.J., and M.P. Huijser. 1999. Mortality factors affecting wild hedgehogs: A study of records from wildlife rescue centres. Lutra 42: 7-24.

Robinson, I., and A. Routh. 1999. Veterinary care of the hedgehog. Practice 21: 128-137.

Roos, S., A. Johnston, and D. Noble. 2012. UK Hedgehog datasets and their potential for long-term monitoring. BTO Research Report No. 598, 1-63 Thetford, British Trust for Ornithology.

RSPCA. 2013. Wildlife Rehabilitation Protocol, Hedgehogs.

Sainsbury, A.W., A.A. Cunningham, P.A. Morris, J.K. Kirkwood, and S.K. Macgregor. 1996. Health and welfare of rehabilitated juvenile hedgehogs (Erinaceus europaeus) before and after release into the wild. Veterinary Record 138: 61-65.

Stocker, L. 2013. Practical wildlife care. New York: Wiley.

Warwick, H., P. Morris, and D. Walker. 2006. Survival and weight changes of hedgehogs (Erinaceus europaeus) translocated from the Hebrides to Mainland Scotland. Lutra 49: 89-102.

Wembridge, D.E., M.R. Newman, P.W. Bright, and P.A. Morris. 2016. An estimate of the annual number of hedgehog (Erinaceus europaeus) road casualties in Great Britain. Mammal Сoтmunications 2: 8-14.

Wilson, E., and D. Wembridge. 2018. The state of Britain's Hedgehogs 2018. London: People's Trust for Endangered Species.

Yarnell, R.W., J. Surgey, A. Grogan, R. Thompson, K. Davies, C. Kimbrough, and D.M. Scott. 2019. Should rehabilitated hedgehogs be released in winter? A comparison of survival, nest use and weight change in wild and rescued animals. European Journal of Wildlife Research 65: 6.

Young, R.P., J. Davison, I.D. Trewby, G.J. Wilson, R.J. Delahay, and C.P. Doncaster. 2006. Abundance of hedgehogs (Erinaceus europaeus) in relation to the density and distribution of badgers (Meles meles). Journal of Zoology 269(3): 349-356.

Publisher's Note Springer Nature remains neutral with regard to jurisdictional claims in published maps and institutional affiliations. 